\title{
In this issue
}

By John Ashkenas, Science Editor

\section{Integrins and signaling in psoriasis}

(See article on pages 527-536.)

The development of keratinocytes in the skin provides one of the clearest examples of a regulated change in cellular adhesion. Basal stem cells and their immediate progeny, the pluripotent "transit" cells that provide a continuous supply of new skin cells, are more highly adhesive than are terminally differentiated suprabasal cells, and they express correspondingly higher levels of several integrins. This regulation is lost in psoriatic lesions, which maintain integrin expression even at the suprabasal level. Following up on earlier evidence that suprabasal expression of integrins in mice is sufficient to induce a hyperproliferation and histological abnormalities that resemble psoriasis, Haase et al. have now sought evidence of signaling abnormalities that might explain the effect of these receptors. The authors focus on the MAP kinase pathway, which mediates integrin effects on basal cell growth. They show here that they can bypass a requirement for high integrin levels by using a constitutively active version of the regulatory MAP kinase kinase MEK to maintain high MAP kinase activity in suprabasal cells, thus promoting keratinocyte growth. Analysis of these cells maintained in organ culture suggests that this transgene delays terminal differentiation by transit cells and generates a thickened basal layer much like that seen in psoriasis. Because an inhibitory form of MEK opposes keratinocyte proliferation in this system, the authors suggest that MAP kinase would be a suitable target for drug therapies for this common disorder.

\section{Fatal thrombotic disease in designer mice lacking vascular thrombomodulin}

(See article on pages 537-546.)

The protein $C$ pathway, which is initiated by the interaction of thrombin with the vascular surface protein thrombomodulin (TM), provides an important brake on blood clotting. Although defects in protein $\mathrm{C}$, protein $\mathrm{S}$, and clotting factor $\mathrm{V}$, other players in this pathway, are known causes of thrombosis, the physiological role of TM in adult hemostasis has proven difficult to pin down, in part because TM serves an early, essential role in development. Indeed, Weiler and his colleagues have found that deletion of the Tm gene in mice causes early death of embryos and that the critical site of this protein's developmental function is not the blood vessel endothelium, but the placenta. Now the same group has used the Cre/LoxP system to generate mice lacking the $T m$ gene specifically in endothelial cells. Many of these mice survive gestation but develop flagrant thrombosis in early adulthood and eventually die with a consumptive coagulopathy. Continuous low-dose exposure to the coagulation inhibitor warfarin prevents these symptoms and allows mice to survive despite the lack of TM, confirming that the disease is one of uncontrolled thrombosis. Males and females show different rates of disease progression in this strain, but the basis of this effect and the possible relation with the coincidence of disease and sexual maturity remain mysterious.

\section{Posttraumatic vaccination for spinal cord injury}

(See article on pages 591-599.)

Here Hauben and coworkers explore a remarkable strategy by which animals may be vaccinated against the effects of spinal cord injury. While vaccination could hardly be expected to prevent a physical trauma, the authors note that much of the tissue destruction and nerve death that leads to paralysis following mild trauma to the spine occurs a week or more after injury and is mediated by immune cells. Building on their previous observation that exogenous $\mathrm{T}$ cells specific for CNS self antigens can block these harmful responses, Hauben et al. have tested whether vaccination with CNS proteins or peptides can activate endogenous $T$ cells to provide mice a similar protective autoimmunity. Such a strategy walks a knife's edge,

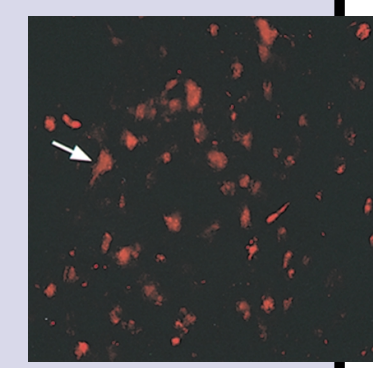
since similar treatments in some mouse strains are used to induce experimental autoimmune encephelitis (EAE), a model of multiple sclerosis. The possibility that a similar pathological response could occur, perhaps variably, in humans, would clearly need to be addressed before this approach could be considered for the clinic. Nevertheless, Hauben et al. show here that one well-studied variant of a myelin basic protein epitope can be used in either EAE-prone or EAE-insensitive mouse strains to promote protective autoimmune responses and prevent the paralysis that would otherwise occur following injury. 